\title{
Towards a mechanically-rechargeable solid fuel flow battery based on earth-abundant materials
}

\author{
Alexis M. Fenton Jr. ${ }^{a, \|}$, Yasser Ashraf Gandomi ${ }^{a, \|}$, Christopher T. Mallia ${ }^{\mathrm{b}}$, \\ Bertrand J. Neyhouse ${ }^{\mathrm{a}}$, M. Aba Kpeglo ${ }^{\mathrm{a}}$, William E. Exson ${ }^{\mathrm{a}}$, Fikile R. Brushett ${ }^{\mathrm{a}, \mathrm{z}}$ \\ ${ }^{a}$ Department of Chemical Engineering, Massachusetts Institute of Technology, 77 Massachusetts \\ Avenue, Cambridge, MA 02139, USA \\ ${ }^{b}$ Department of Material Science and Engineering, Massachusetts Institute of Technology, 77 \\ Massachusetts Avenue, Cambridge, MA 02139, USA \\ " Equal author contribution \\ ${ }^{z}$ Corresponding author(F.R. Brushett, +16173247400, brushett@mit.edu)
}

\begin{abstract}
:
Metal-air batteries are a promising energy storage solution, but materials limitations (e.g., metal passivation, low active material utilization) have stymied their adoption. We investigate a solid fuel flow battery (SFFB) architecture that combines the energy density of metal-air batteries with the modularity of redox flow batteries. Specifically, a metallic solid electrochemical fuel (SEF) is spatially separated from the anodic current collector; a dissolved redox mediator shuttles charge between the two, and an oxygen reduction cathode completes the circuit. This modification decouples power and energy system components while enabling mechanical rechargeability and mitigating the effects of non-uniform metal oxidation. We conduct an exploratory study showing that metallic SEFs can chemically reduce organic redox mediators repeatedly. We subsequently operate a proof-of-concept SFFB cell for ca. 25 days as an initial demonstration of technical feasibility. Overall, this work illustrates the potential of this storage concept and highlights scientific and engineering pathways to improvement.
\end{abstract}

Table of Contents Image:
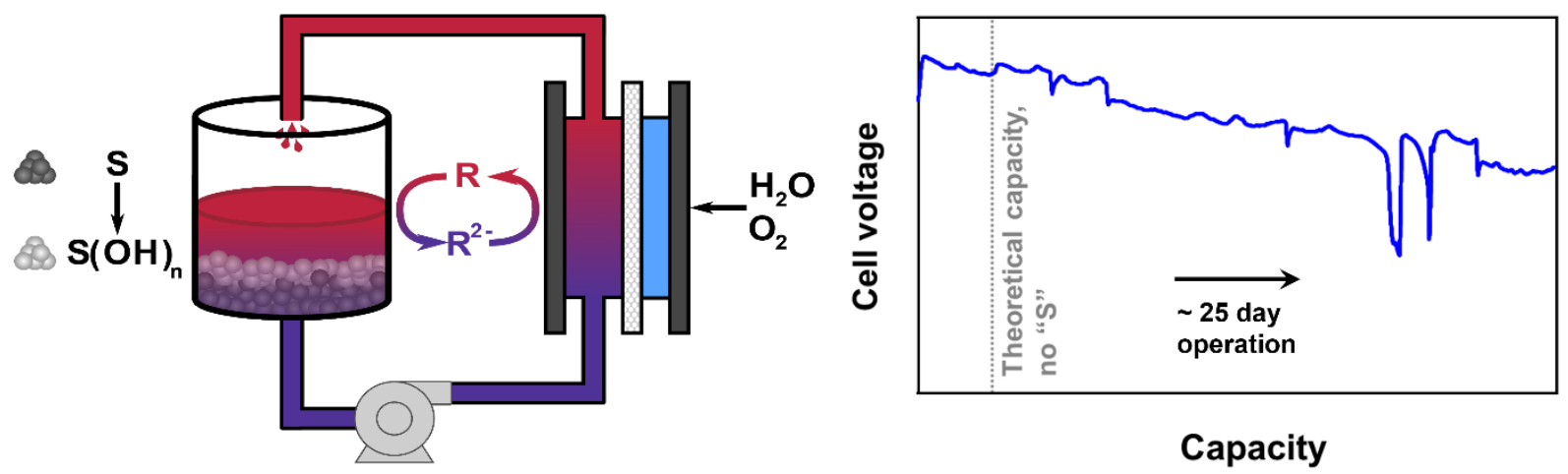
Electrochemical energy storage is anticipated to be an important component of a sustainable energy economy, and the continued development of rechargeable battery technologies is key to global decarbonization efforts. ${ }^{1,2}$ While numerous rechargeable batteries have been developed, lithium-ion (Li-ion) batteries represent the current state-of-the-art due to their favorable combination of power density, efficiency, and cycle life. However, the energy density, cost, and scale requirements of emerging applications (e.g., heavy-duty transportation, long-duration storage) challenge current Li-ion battery technologies, motivating research and development into a diverse array of redox chemistries and system formats that may better suit current and future societal needs.

Metal-air batteries have attracted considerable attention over the years due in part to the high charge-storage capacity of metallic negative electrodes - along with the low cost and high terrestrial abundance of oxygen $\left(\mathrm{O}_{2}\right)$ - which, taken together, offer a pathway to inexpensive and energy dense storage. ${ }^{3-5}$ While these batteries have found success as primary (non-rechargeable) applications (e.g., hearing aids), deployment of rechargeable batteries at large scales has generally been hampered by a combination of low active material utilization, limited cycle life, and poor roundtrip energy efficiency. ${ }^{3}$ Herein, we explore opportunities to enable more versatile operation by mitigating some of these limitations through an alternative cell design: the solid fuel flow battery (SFFB). Specifically, instead of utilizing a conventional enclosed cell typical of most metal-air batteries, the SFFB adopts a hybrid architecture more akin to that of redox flow batteries (RFBs) and direct liquid fuel cells, where a metallic solid electrochemical fuel (SEF) is housed in an external tank spatially separated from the electrochemical reactor. A solubilized redox mediator (RM) transports charge between the SEF and the anodic current collector (Figure 1), and the cathodic $\mathrm{O}_{2}$ reduction reaction (ORR) completes the electrical and ionic circuits. Such spatial 
decoupling eliminates the need to maintain electrical contact between the current collector and the metal, ostensibly reducing the detrimental effects of passivation and lowering the materials processing requirements (e.g., metallic-film or composite electrodes) —in turn potentially improving cell performance (i.e., materials utilization). Further, charging options are expanded to include mechanical recharging via facile periodic SEF exchange, circumventing the challenges of metal electrodeposition at high rates and long durations. This architecture may also enable multiple different SEF-RM pairings to be used depending on installation site, materials availability, or process economics. Overall, these benefits make the SFFB a promising energy storage concept with potentially increased versatility in material composition, modification, and maintenance.

In recent years, there have been several reports that describe electrochemical processes that use solubilized RMs to transfer charge between physically separated reactants and an electrode for the purpose of energy storage and conversion..$^{6-9}$ Wang and coworkers have pioneered rechargeable mediated flow batteries with solid active materials in external tanks via redoxtargeting - the process of selecting or engineering RMs by modulating their redox potential to favorably exchange electrons with a solid (insoluble) material. ${ }^{6,7}$ Deng and coworkers have also demonstrated a fuel cell targeting biomass byproducts of pulp and paper processing using iron (Fe)-based mediators, while Stahl and coworkers have extensively studied mediated fuel cell architectures with both organic and inorganic redox chemistries.${ }^{8,9}$ Here, we explore the feasibility of implementing metallic SEFs in modified metal-air systems, with a particular focus on RM-SEF interactions in the external storage tank (Figure 1). We first use in situ microelectrode voltammetry, post mortem X-ray diffractometry, and post mortem optical microscopy to assess the efficacy of RM-SEF pairings-identifying 2-hydroxynaphthoquinone (2-HNQ) and zinc (Zn) as a favorable combination. We then assemble a proof-of-concept cell and demonstrate galvanostatic 
operation over $c a .25$ days, discharging $c a .30 \%$ of the $\mathrm{Zn}$ in the external tank. Overall, this work seeks to articulate foundational design principles of and experimental methods for SFFBs and aims to inspire future studies that expand upon this concept.
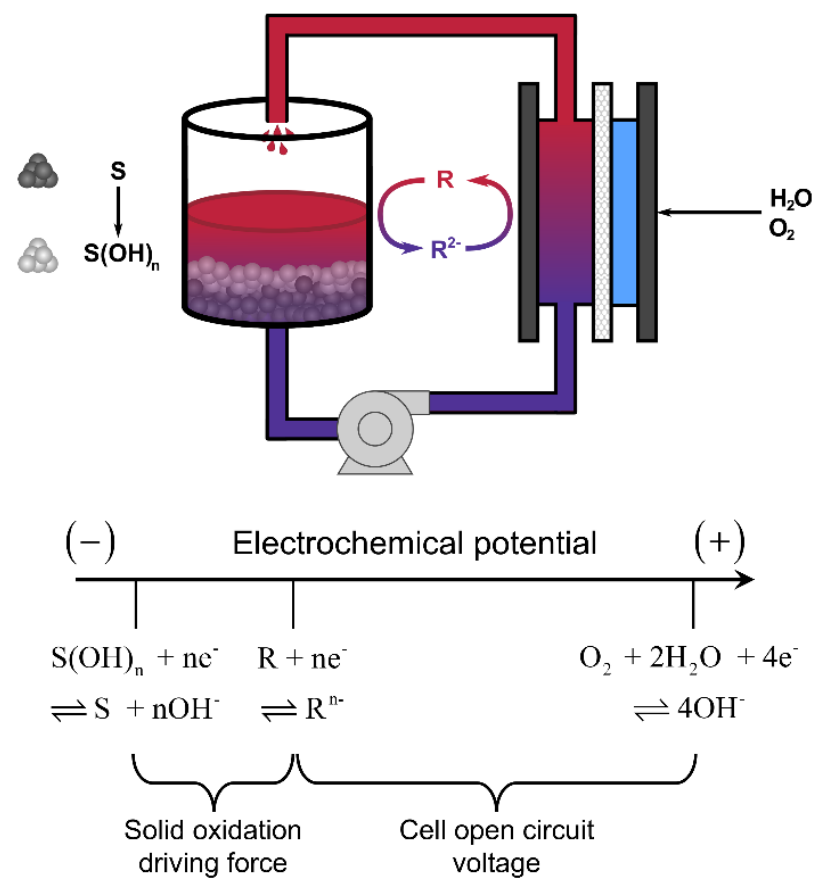

Figure 1. Schematic illustration of the SFFB. In the cathode, $\mathrm{O}_{2}$ and water react to form hydroxyl ions, which cross the membrane to the anodic half-cell. There, the reduced $R M$ " $R$ "-" is oxidized to R, which, along with the hydroxyl ions, are subsequently pumped to the storage tank, where the SEF ("S") oxidizes as it chemically reduces $R$ to $\mathrm{R}^{2-}$. The recharged $\mathrm{R}^{2-}$ is then pumped to the anode to be re-oxidized. Below the diagram, the reactions are shown on an electrochemical potential schematic; relative potential spacings are for conceptual illustration and are not drawn to scale.

\section{Materials selection criteria}

The first criterion for successfully operating a SFFB is a suitable SEF and RM combination.

As illustrated in Figure 1, there must be a sufficient thermodynamic driving force between the SEF and the RM to promote the chemical redox reaction, yet too great a driving force lowers the 
cell voltage and thus the accessible power and energy density. Parasitic side reactions (e.g., hydrogen evolution) must also be mitigated. We selected $\mathrm{Zn}$ and Fe as candidate SEFs and 2-HNQ and 2,6-dihydroxyanthraquinone (2,6-DHAQ) as candidate RMs. Fe and $\mathrm{Zn}$ are earth-abundant metals with extensive histories as anodes in aqueous metal-air battery literature, while 2-HNQ and 2,6-DHAQ have recently been demonstrated in aqueous organic RFBs. ${ }^{10-13}$ To enable greater materials compatibility, to reduce the propensity for hydrogen generation, and to close the supporting ion balance, we investigated cell operation in an alkaline environment. We explored all four combinations of SEFs and RMs; only the results for the best-performing pair- $\mathrm{Zn}$ and 2HNQ - are described in the main text, while those of other pairings are found in the Supplementary Information (SI).

\section{Estimating the chemical reduction of RMs using microelectrode voltammetry}

While the electrode reactions occurring within the flow cell (ORR and quinone oxidation) are reasonably well-understood, ${ }^{12,14}$ the redox reactions between the RM and SEF have not been as extensively studied. As such, we sought to assess whether the selected metals can chemically reduce the solubilized RMs. We selected SEFs and RMs with sufficiently disparate electrode potentials to enable a suitable thermodynamic driving force - for example, Zn oxidizes at $\sim-1.2$ $\mathrm{V}$ vs. SHE, and 2-HNQ has an observed redox potential of $\sim-0.5 \mathrm{~V}$ vs. SHE, resulting in a $c a$. $700 \mathrm{mV}$ overpotential. To measure the extent of the reaction, we used a three-electrode configuration to monitor the time-dependent changes in the RM state-of-charge (SOC) in the presence of a stoichiometric excess of SEF. Specifically, we used microelectrode voltammetry, where the small working electrode radius $(c a .1-10 \mu \mathrm{m})$ produces steady-state currents that simultaneously describe the charged and discharged active species concentrations, which, in turn, can be processed to estimate the solution SOC and state-of-health (SOH). ${ }^{15-17}$ 
Figure 2a shows representative microelectrode voltammograms acquired on a carbon fiber working electrode throughout the experiment, depicting changes in the steady-state current plateaus representative of the time-dependent SOC. To increase the rate of $\mathrm{Zn} / 2$-HNQ mixing and potentially the rate and extent of the chemical redox reaction at the upper limits of SOC, sparging was more aggressively conducted starting at $70 \mathrm{~min}$; the stir rate, in turn, was increased from 600 to $1400 \mathrm{rpm}$ at $80 \mathrm{~min}$ for the same purpose. Multiple independent trials $(\mathrm{n}>3)$ were conducted; 2-HNQ was consistently reduced in these trials, supporting the data shown for the individual trial in Figure 2. We calculate the SOC (Figure 2b) by assuming a diffusion coefficient ratio of unity $-D_{\text {Red }} \cdot D_{O x}^{-1}=1$, where $D_{\text {Red } / O x}\left(\mathrm{~m}^{2} \mathrm{~s}^{-1}\right)$ refer to the respective diffusion coefficients of the reduced and oxidized forms of a generic redox couple ${ }^{18}$ - but we also consider differing ratios $\left(D_{R e d} \cdot D_{O x}^{-1}=0.5\right.$ and $\left.D_{R e d} \cdot D_{O x}^{-1}=2\right)$ to respectively serve as upper and lower SOC bounds. This analysis enables a general trend to be discerned beyond the specific redox couples examined; we find that minor variations in this ratio do not significantly alter SOC predictions.

The Zn / 2-HNQ electrolyte approaches $95 \%$ SOC from an entirely discharged state (i.e., nearcomplete 2-HNQ reduction) within $2 \mathrm{~h}$. Concurrently, the magnitude of the sigmoid wave (the apparent $\mathrm{SOH}$ ) decreases by $c a .20 \%$, which is potentially due to molecular decay but may also be the result of other phenomena, such as a non-unity $\left(D_{R e d} \cdot D_{O x}^{-1} \sim 0.8\right)$ diffusion coefficient ratio within the bounds of Figure 2b. The other candidate RM / SEF pairs display significantly lower conversions over similar timescales, which we tentatively attribute to surface passivation. These semi-quantitative studies are intended to evaluate whether charge-transfer reactions occur between the RM and the SEF at appreciable rates; the development of microscopic rate laws and quantitative extensions to reactor-scale experiments and models require more comprehensive analyses beyond the scope of this initial report. 

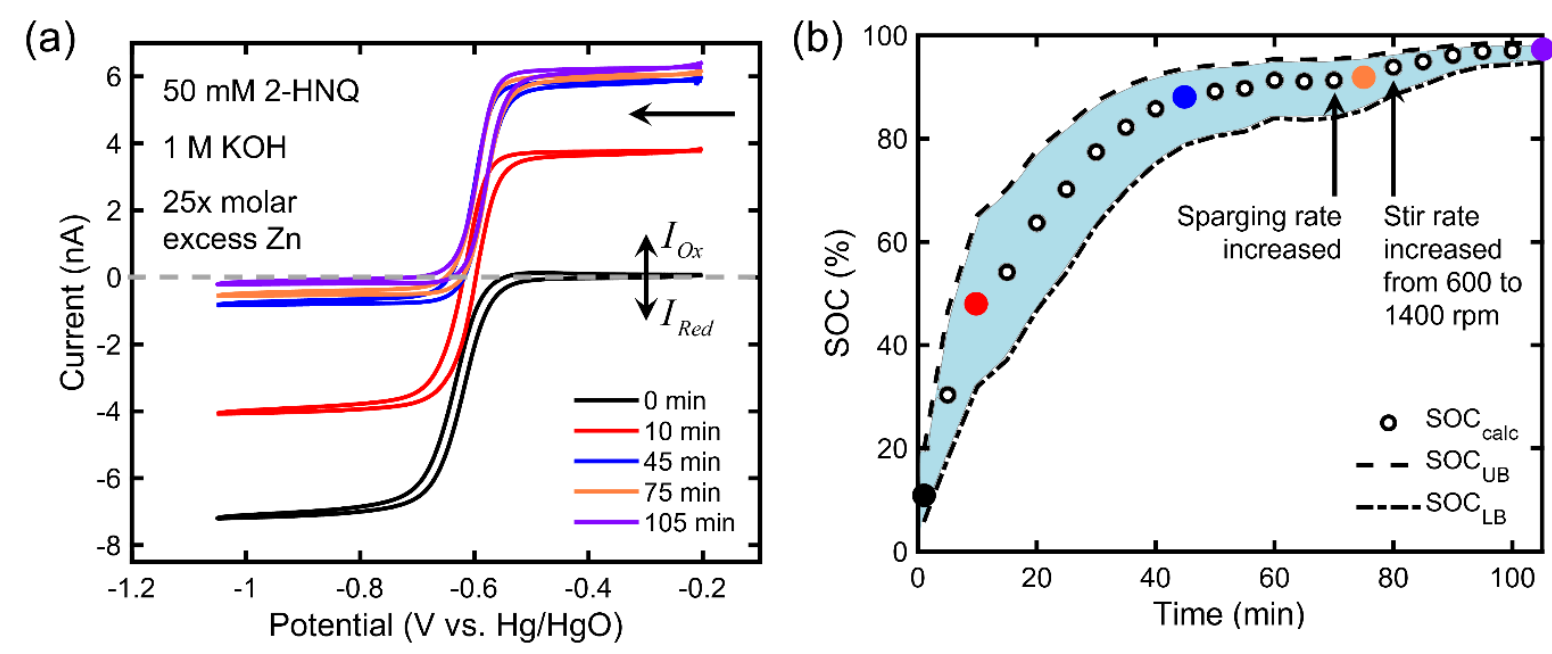

Figure 2. (a) Example microelectrode voltammograms from a representative dataset where 2HNQ is charged with $\mathrm{Zn}$. The initial sweep direction is reductive (via the horizontal arrow); positive currents are oxidative, and negative currents are reductive. (b) The variation in the SOC at various times. $D_{R e d} \cdot D_{O x}^{-1}$ was assumed to be 1 ; the upper and lower bound estimates, SOCUB/LB, respectively refer to $D_{R e d} \cdot D_{O x}^{-1}=0.5$ and 2 , with the range shaded in light blue. The filled circles correspond to the voltammograms in (a), and the sparging and stir rates were both increased to promote solution agitation.

Estimating the chemical oxidation of SEFs via X-ray diffraction and optical microscopy

While the prior experiments indicate 2-HNQ is reduced in the presence of $\mathrm{Zn}$, it is unclear whether $\mathrm{Zn}$ is the dominant electron source. As such, we performed a combination of X-ray diffraction and optical microscopy to explore the evolution of the $\mathrm{Zn} \mathrm{SEF}$ in Figure 3. 


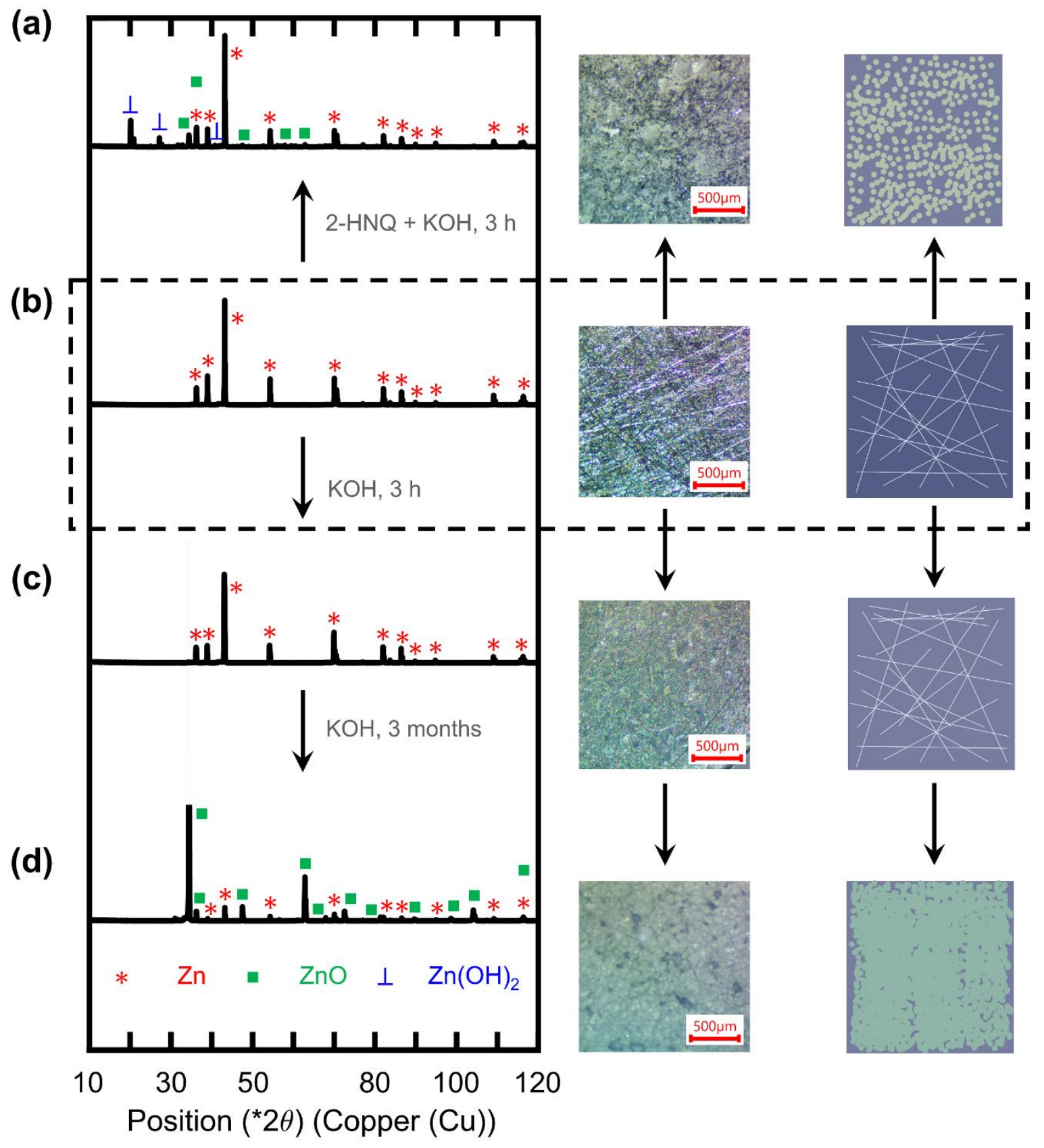

Figure 3. Diffractograms (left column), optical micrographs (center column), and illustrative diagrams (right column) of $\mathrm{Zn}$ oxidation in $1 \mathrm{M}$ potassium hydroxide $(\mathrm{KOH})$ with and without 50 $\mathrm{mM}$ 2-HNQ. (a) Post mortem observations of $\mathrm{Zn}$ after residing in an aqueous solution of $50 \mathrm{mM}$ 2-HNQ and $1 \mathrm{M} \mathrm{KOH}$ for $3 \mathrm{~h}$. (b) Analogous observations for pristine, unreacted $\mathrm{Zn}$. (c) Observations for $\mathrm{Zn}$ after residing in $1 \mathrm{M} \mathrm{KOH}$ (no 2-HNQ) for $3 \mathrm{~h}$. (d) Observations for $\mathrm{Zn}$ after residing in $1 \mathrm{M} \mathrm{KOH}$ (no 2-HNQ) for 3 months. Vertically stacked symbols indicate multiple species contributed to the observed diffractogram peak. In part (d), the larger ZnO peak near a $2 \theta$ of $35^{\circ}$ was truncated to improve visibility. Within the illustrative diagrams, the dark and faded 
violet backgrounds respectively represent (un)tarnished $\mathrm{Zn}$, the silver lines represent striations on the as-received $\mathrm{Zn}$, and circles represent solid deposits. More detailed diffraction results are provided in the SI.

Figure 3b depicts the baseline diffractogram (left column), optical micrograph (center column), and associated illustration (right column) of $\mathrm{Zn}$ foil unexposed to the solution. Figure 3a indicates that $\mathrm{ZnO}$ and $\mathrm{Zn}(\mathrm{OH})_{2}$ are present with the $\mathrm{Zn}$ after it resides in an aqueous solution of $50 \mathrm{mM} 2$ HNQ and $1 \mathrm{M}$ potassium hydroxide $(\mathrm{KOH})$ for $3 \mathrm{~h}$. Figures $3 \mathbf{c}$ and $\mathbf{3 d}$, in turn, indicate that though Zn oxidizes in a solution containing only water and $1 \mathrm{M} \mathrm{KOH}$ (no 2-HNQ), the reaction proceeds more slowly and possibly via different mechanisms- $\mathrm{Zn}(\mathrm{OH})_{2}$ is not observed-suggesting $\mathrm{Zn}$ contributes towards reducing 2-HNQ. These findings generally agree with the current understanding of $\mathrm{Zn}$ oxidation in alkaline conditions, which can proceed through multiple pathways. ${ }^{11,19-22}$ A passivation layer may form but is thought to be porous, ${ }^{23}$ allowing for reduced but finite mass transfer of 2-HNQ to the buried $\mathrm{Zn}$ surface to continue the chemical redox reaction. Hydrogen evolution also occurs in the presence of $\mathrm{Zn}$ in alkaline solutions, though it appears to be slower than 2-HNQ reduction, as evinced by Figures 3a and 3c. These analyses indicate that 2$\mathrm{HNQ}$ is reduced in the electrolyte due primarily to its interactions with $\mathrm{Zn}$, which, in turn, is corroded.

\section{Proof-of-concept demonstration with a hybrid flow cell architecture}

To explore the efficacy of the charge transfer between the SEF and RMs on a device level, proof-of-concept experiments were conducted with the 2-HNQ / Zn pairing using a flow cell architecture (Figure 4a) adopted from a prior design modified to enable the ORR via a silvercoated gas diffusion electrode. ${ }^{24}$ To ensure sufficient 2-HNQ charging from the $\mathrm{Zn}$ and adequate electrode wetting, we conduct open circuit voltage (OCV) studies, which should increase as the 2- 
HNQ charges according to the Nernst equation. Indeed, the OCV increases throughout the experiment; however, the maximum measured voltage $(\sim 0.68 \mathrm{~V})$ was lower than the theoretical $(\sim 0.9 \mathrm{~V})$ by $c a .200 \mathrm{mV}$, possibly due to reactant crossover causing mixed electrode potentials and / or catalyst poisoning. ${ }^{25}$

To demonstrate repeatable performance and to explore the behavior of the reactor at different discharge conditions, we assembled a second cell operated at various current densities up to $c a .10$ $\mathrm{mA} \mathrm{cm}{ }^{-2}$. With sufficient electrolyte flow $\left(\geq 10 \mathrm{~mL} \mathrm{~min}^{-1}\right)$, the polarization behavior was largely insensitive to $\mathrm{O}_{2}$ flow rate (range $2-20 \mathrm{~mL} \mathrm{~min}^{-1}$ ). Encouraged by these findings, we conducted a longer duration discharge experiment at an electrolyte flow rate of $10 \mathrm{~mL} \mathrm{~min}^{-1}$, an $\mathrm{O}_{2}$ flow rate

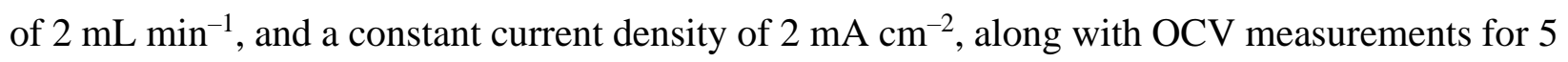
min every hour (not shown). As shown in Figures $\mathbf{4 b}$ and $\mathbf{c}$, the cell delivered an average voltage of $c a .0 .38 \mathrm{~V}$ that gradually declined during the extended discharge period. During this time, the OCV similarly declined from $0.61 \mathrm{~V}$ to $0.5 \mathrm{~V}$, indicating that polarization was not the only factor limiting performance. We posit that the cause of this decay was material depletion via undesired water crossover to and evaporation from the cathode or other leak points in the testing apparatus. 2-HNQ may also undergo chemical homogeneous or heterogeneous decomposition in the anodic half-cell, with the reduced mediator concentrations potentially leading to mass transport limitations that lower the cell voltage during operation. ${ }^{12}$ To allow the cell voltage to partially recover, the cell discharge was occasionally stopped for longer periods ( $>5 \mathrm{~min}$ )—referred to as "pauses" - and the electrolyte reservoir was sometimes refilled (respectively denoted by the black and red circles in Figure 4b); successful voltage recovery after pauses indicates the tank reaction may be rate-limiting in this device. The longevity of the cell suggests that performance degradation was not rapid or could otherwise be mitigated; indeed, the experiment ended due to equipment 
needs rather than cell death. Overall, $c a .30 \%(1.725 \mathrm{Ah})$ of the theoretical capacity from the $\mathrm{Zn}$ was accessed during $c a .25$ days of galvanostatic discharge (Figure 4c). 
(a)

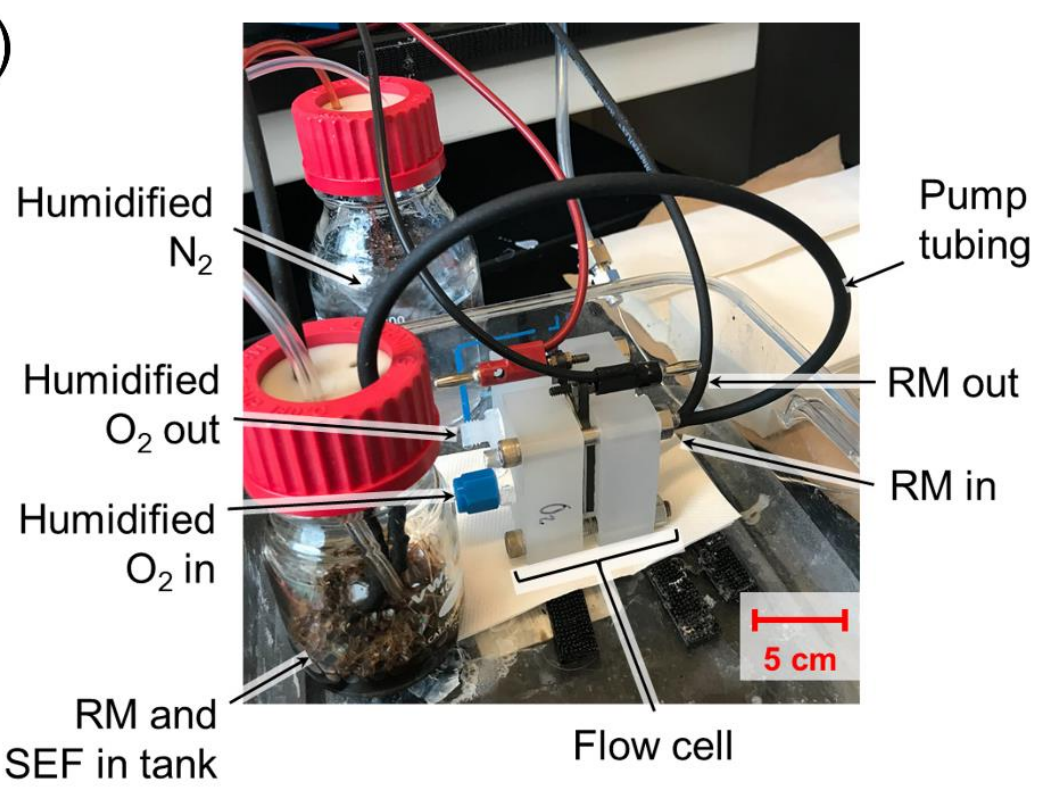

(b)

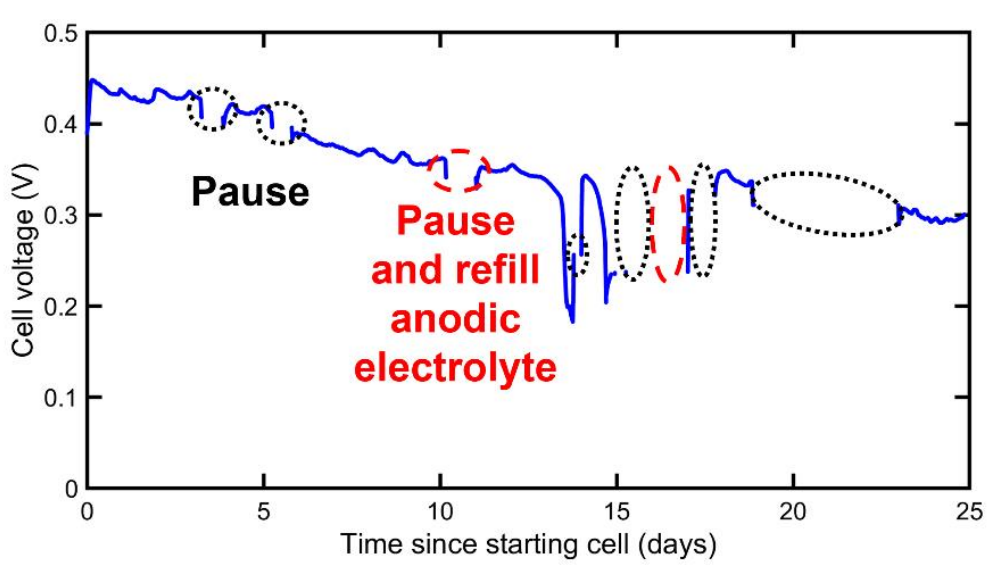

(c)

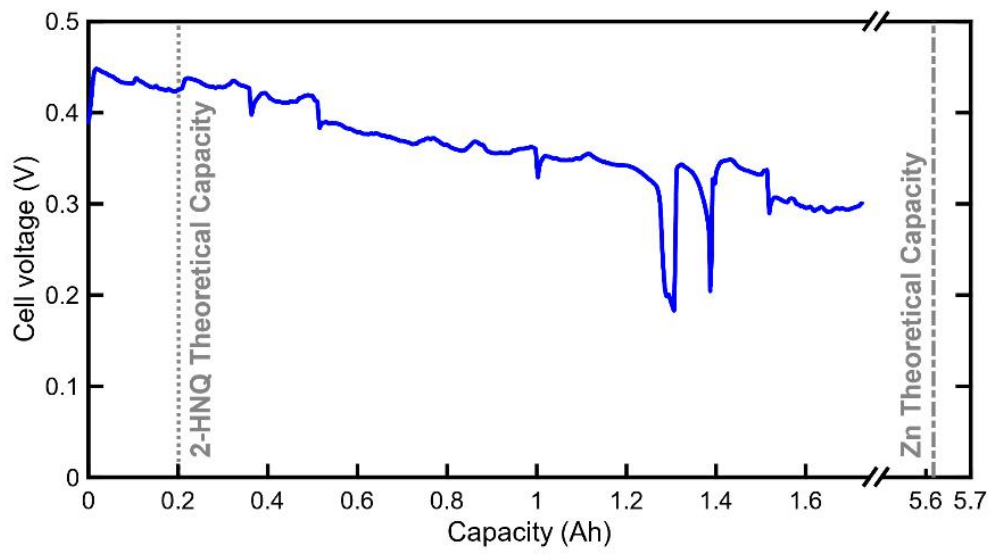

Figure 4. (a) Labeled photograph of the assembled SFFB used for OCV monitoring; the scale bar is qualitative. (b) Flow cell discharge, with outliers and smoothing processed using $1 \mathrm{~h}$ moving 
median and mean windows, respectively. Extended pauses in the galvanostatic discharge were taken (black or red circles), sometimes to refill the anodic electrolyte volume (red circles). (c) The same discharge curve as (b) plotted versus capacity.

In this letter, we explore an energy storage concept — a SFFB — that seeks to combine the high energy density of a metal-air battery with the architecture of a redox flow battery. Using a combination of electrochemical, X-ray diffraction, and optical microscopy methods, we identified 2-HNQ and $\mathrm{Zn}$ as a promising material set. Finally, we demonstrated the feasibility of this approach using a proof-of-concept flow cell setup which operated for $c a$. 25 days, accessing $c a$. $30 \%$ of the theoretical capacity of the $\mathrm{Zn}$ in the reservoir.

While these exploratory studies suggest that the SFFB may hold promise as a modular and energy-dense device, further studies are needed to understand the potential of this storage approach. Microscopic analyses of the rates and selectivity of the charge-transfer reactions between the soluble mediator and solid fuel are needed to articulate reaction rate laws and to identify opportunities to enhance performance, accessible capacity, and longevity through material selection and electrolyte engineering. Of particular importance is the role of the thermodynamic driving force and evolving interfacial chemistry on observed reaction rates. Further, refinements in membrane-electrode assembly engineering may enable improvements in operating cell potential and current density through reductions in cell resistance, increases in catalytic / electrode activity, and mitigation of mediator crossover and water evaporation. Finally, system design and engineering efforts are needed to correctly size the tank and electrochemical reactor to maximize overall performance. 


\section{Experimental Methods}

$\underline{\text { Materials and reagents }}$

2-HNQ (Thermo Scientific ${ }^{\mathrm{TM}}$, AAA1188009, 98+ \%) and KOH (Sigma-Aldrich ${ }^{\circledR}, \mathrm{P} 1767, \geq$ $85 \%$ ) were used as received. Zn foil of $0.25 \mathrm{~mm}$ thickness (Thermo Scientific ${ }^{\mathrm{TM}}$, AA10436BW, $99.98 \%$ ) was hand-cut with scissors into smaller pieces (each $\mathrm{ca} .1-5 \mathrm{~cm}^{2}$ ) before being added to the solution containing the RM. Details on other RM-SEF pairings are provided in the SI.

\section{$\underline{\text { Microelectrode experiments }}$}

The electrolyte solutions tested consisted of $50 \mathrm{mM}$ 2-HNQ and $1 \mathrm{M} \mathrm{KOH}$ dissolved in deionized water $(18.2 \mathrm{M} \Omega \mathrm{cm})$ equipped with a carbon fiber microelectrode (Bioanalytical Systems, Inc. ${ }^{\circledR}\left(\mathrm{BASi}^{\circledR}\right), \mathrm{MF}-2007,8-13 \mu \mathrm{m}$ nominal diameter range); a platinum(Pt) coil (BASi ${ }^{\circledR}$, $\mathrm{MW}-1033)$, wire $\left(\mathrm{BASi}^{\circledR}, \mathrm{MW}-1032\right)$, or mesh counter electrode; and a $\mathrm{Hg} / \mathrm{HgO}$ reference electrode (BASi ${ }^{\circledR}$, EF-1369). Polishing was conducted using $0.05 \mu \mathrm{m}$ alumina powder (Buehler ${ }^{\circledR}$, MicroPolish Powder, 4010075) in deionized water. To remove residual $\mathrm{O}_{2}$, the system was initially sparged for 10 min with $\mathrm{N}_{2}$ (Airgas, $99.999 \%$ ) humidified in-house. An initial (set of) voltammogram(s) was acquired before addition of the Zn, after which 25 molar equivalents ( $c a$. $0.82 \mathrm{~g}$ ) of the $\mathrm{Zn}$ foil were added to chemically reduce $2-\mathrm{HNQ}$. We then recorded microelectrode voltammograms at various time intervals - typically $5 \mathrm{~min}$ - to monitor the RM SOC at room temperature $\left(20-25^{\circ} \mathrm{C}\right)$. A stir bar $\left(\mathrm{VWR}^{\circledR}, 58948-375\right)$ was also used to promote mixing; during voltammetric acquisition, the stir bar was stopped, and the sparging line was raised above the electrolyte, to enable solution quiescence. To increase the chemical redox reaction rate, both the rate of sparging and stirring were occasionally increased. 


\section{$\underline{\text { X-ray diffractometry experiments }}$}

X-ray diffractograms of Zn were acquired using a PANalytical X'Pert PRO XRPD (1.8 kW sealed X-ray tube source with a copper target and a vertical circle theta:theta goniometer, $240 \mathrm{~mm}$ radius). PANalytical HighScore Plus was used to regress the constituent phases from the diffractograms to a library referenced from the International Center for Diffraction Data PDF database.

\section{Optical microscopy experiments}

Optical microscopy was performed on an AmScope 7-45 $\times$ magnification industrial inspection microscope equipped with a 9.0 MP USB camera and $20 \times / 10$ eyepieces (AmScope). Software provided by AmScope was used for image acquisition, with scale calibrated with an external optical reference micrometer scale. LED top-down illumination was used for opaque metal samples, and focusing was manually adjusted for each magnification level. Samples were imaged as-extracted after experiments, subsequently rinsed in DI water for 30 seconds, and imaged again. All expended metal samples were stored in HDPE scintillation vials (VWR).

\section{$\underline{\text { Full cell studies }}$}

Two separate cells were studied for repeatability — one for an OCV analysis and another for polarization and long-term operation studies. Major components included graphite interdigitated flow fields, porous electrodes, an anion-exchange membrane (Membranes International Inc., AMI7001S), and plastic backing plates. The anode was a carbon paper (Sigracet 29AA) electrode thermally treated in air at $400{ }^{\circ} \mathrm{C}$ for $30 \mathrm{~h}$ to improve aqueous electrolyte wetting. The cathode was a hydrophobically-treated carbon paper gas diffusion electrode (Freudenberg H23C6, Fuel Cell Store) with a silver (Ag)-nanoparticle-based catalyst layer spray coated onto the microporous layer; the catalyst-coated side of the cathode was abutted to the membrane to enable ionic 
connection. Both electrodes were compressed to $\sim 75 \%$ of their nominal thickness to provide sufficient conductivity and reduce contact resistances.

Further, a Masterflex ${ }^{\circledR}$ peristaltic pump (Cole Parmer) with Masterflex ${ }^{\circledR}$ Norprene $^{\circledR}$ tubing (Size: L/S 14, Cole Parmer) was used to circulate the electrolyte. The electrolyte flowed through the anodic half-cell at various flow rates, and high purity $\mathrm{O}_{2}$ (Airgas, OX UHP300) was passed through the cathode. The setup also included the gas flow controllers (Brooks Instruments) and an in-house gas humidification unit to respectively control the $\mathrm{O}_{2}$ flow rate and humidify the inlet gas in ambient temperature; the humidity level was not quantified throughout the experiment but is estimated to be $\sim 20 \mathrm{wt} \%$ based on thermodynamic calculations. A NPT-type fitting was used to connect the oxygen line to the reactor. For the anodic side, the storage tank on the anode side was a $100 \mathrm{~mL}$ (for the first cell) or $250 \mathrm{~mL}$ (for the second cell) glass media bottle which contained the aqueous electrolyte (50 mM 2-HNQ dissolved in $1 \mathrm{M} \mathrm{KOH})$ and initially ca. $1.8 \mathrm{~g}$ of the hand-cut Zn foil. The tank was sealed using a rubber septum with four holes punched—two 3/16" holes for the electrolyte inlet / outlet, one 3/16" hole for the sparging line $\left(\mathrm{N}_{2}\right.$ humidified in-house), and one uncovered $1 / 8$ " or $3 / 16 "$ hole to prevent overpressure.

All cells were operated at room temperature $\left(20-25^{\circ} \mathrm{C}\right)$. The $\mathrm{OCV}$ of the first cell that contained $20 \mathrm{~mL}$ of electrolyte in the storage tank was monitored for $c a .12 \mathrm{~h}$. The subsequent cell, in turn, contained $75 \mathrm{~mL}$ of electrolyte and was operated at various current densities up to $c a .10$ $\mathrm{mA} \mathrm{cm}$. This second cell was also operated for a long-duration study at an electrolyte flow rate of $10 \mathrm{~mL} \mathrm{~min}^{-1}$, an $\mathrm{O}_{2}$ flow rate of $2 \mathrm{~mL} \mathrm{~min}^{-1}$, and a constant current density of $2 \mathrm{~mA} \mathrm{~cm}{ }^{-2}$; the OCV was also measured for 5 min every hour. Extra $\mathrm{Zn}$ (prepared as before) was also added for the long-duration cycling to replicate the same stoichiometric excess used in the microelectrode studies_ca. $25 \times$, or $6.85 \mathrm{~g}$ (5.617 Ah capacity), in total. 
Supporting Information Available: Additional details regarding microelectrode voltammetry theory and experiments; a table summarizing results and experimental details for all four material pairings, with microelectrode voltammetry analyses for the 2,6-DHAQ / Zn, 2,6-DHAQ / Fe, and 2-HNQ / Fe pairings; description of cell assembly and catalyst preparation / spray-coating; full cell results for OCV and polarization tests; and raw X-ray diffractograms and tabulated peaks.

\section{Acknowledgements}

This work was funded by the Skoltech - MIT Next Generation Program. YAG, BJN, and FRB gratefully acknowledge support by the Joint Center for Energy Storage Research, an Energy Innovation Hub funded by the U.S. Department of Energy, Office of Science, Basic Energy Sciences. BJN gratefully acknowledges the NSF Graduate Research Fellowship Program under Grant Number 1122374. Any opinion, findings, and conclusions or recommendations expressed in this material are those of the authors and do not necessarily reflect the views of the NSF. CTM gratefully acknowledges support under and awarded by the Department of Defense, Office of Naval Research, through the National Defense Science and Engineering Graduate Fellowship.

\section{CRediT Author Contribution Statement}

A.M.F., Jr. performed the conceptualization, methodology, software, validation, formal analysis, investigation, data curation, writing-original draft, writing-review and editing, and visualization. Y.A.G. performed the conceptualization, methodology, software, validation, formal analysis, investigation, data curation, writing-original draft, writing-review and editing, and visualization. C.T.M. performed the validation, investigation, data curation, writing-original draft, writing-review and editing, and visualization. B.J.N. performed the writing-original draft, 
writing-review and editing, and visualization. M.A.K. performed the validation and investigation. W.E.E. performed the validation and investigation. F.R.B. performed the conceptualization, funding acquisition, project administration, resources, supervision, writing-original draft, writing-review and editing.

\section{Competing Interests and Funding}

The authors declare no competing financial interest. 


\section{References}

(1) Huang, Q.; Li, H.; Grätzel, M.; Wang, Q. Reversible Chemical Delithiation/Lithiation of $\mathrm{LiFePO}_{4}$ : Towards a Redox Flow Lithium-Ion Battery. Phys Chem Chem Phys 2013, 15, $1793-1797$.

(2) Weber, A. Z.; Mench, M. M.; Meyers, J. P.; Ross, P. N.; Gostick, J. T.; Liu, Q. Redox Flow Batteries: A Review. J. Appl. Electrochem. 2011, 41, 1137-1164.

(3) Rahman, Md. A.; Wang, X.; Wen, C. High Energy Density Metal-Air Batteries: A Review. J. Electrochem. Soc. 2013, 160, A1759-A1771.

(4) Mladenova, E.; Slavova, M.; Mihaylova-Dimitrova, E.; Burdin, B.; Abrashev, B.; Krapchanska, M.; Raikova, G.; Vladikova, D. Monolithic Carbon-Free Gas Diffusion Electrodes for Secondary Metal-Air Batteries. J. Electroanal. Chem. 2021, 887, 115112.

(5) Blurton, K. F.; Sammells, A. F. Metal/Air Batteries: Their Status and Potential - A Review. J. Power Sources 1979, 4, 263-279.

(6) Wang, Q.; Zakeeruddin, S. M.; Wang, D.; Exnar, I.; Grätzel, M. Redox Targeting of Insulating Electrode Materials: A New Approach to High-Energy-Density Batteries. Angew. Chem. Int. Ed. 2006, 45, 8197-8200.

(7) Huang, Q.; Yang, J.; Ng, C. B.; Jia, C.; Wang, Q. A Redox Flow Lithium Battery Based on the Redox Targeting Reactions between $\mathrm{LiFePO}_{4}$ and Iodide. Energy Environ. Sci. 2016, 9, 917-921.

(8) Yang, L.; Liu, W.; Zhang, Z.; Du, X.; Gong, J.; Dong, L.; Deng, Y. Hydrogen Evolution from Native Biomass with $\mathrm{Fe}^{3+} / \mathrm{Fe}^{2+}$ Redox Couple Catalyzed Electrolysis. Electrochimica Acta 2017, 246, 1163-1173. 
(9) Anson, C. W.; Stahl, S. S. Mediated Fuel Cells: Soluble Redox Mediators and Their Applications to Electrochemical Reduction of $\mathrm{O}_{2}$ and Oxidation of $\mathrm{H}_{2}$, Alcohols, Biomass, and Complex Fuels. Chem. Rev. 2020, 120, 3749-3786.

(10) McKerracher, R. D.; Ponce de León, C.; Wills, R. G. A.; Shah, A. A.; Walsh, F. C. A Review of the Iron-Air Secondary Bettery for Energy Storage. ChemPlusChem 2015, 80, $323-335$.

(11) Li, Y.; Dai, H. Recent Advances in Zinc-Air Batteries. Chem. Soc. Rev. 2014, 42, 52575275.

(12) Tong, L.; Goulet, M.-A.; Tabor, D. P.; Kerr, E. F.; Porcellinis, D. D.; Fell, E. M.; AspuruGuzik, A.; Gordon, R. G.; Aziz, M. J. Molecular Engineering of an Alkaline Naphthoquinone Flow Battery. ACS Energy Lett. 2019, 4, 1880-1887.

(13) Lin, K.; Chen, Q.; Gerhardt, M. R.; Tong, L.; Kim, S. B.; Eisenach, L.; Valle, A. W.; Hardee, D.; Gordon, R. G.; Aziz, M. J.; et al. Alkaline Quinone Flow Battery. Science 2015, 349, 1529-1532.

(14) O'Hayre, R. P. Fuel Cell Fundamentals; John Wiley \& Sons: Hoboken, NJ, 2016.

(15) Stolze, C.; Meurer, J. P.; Hager, M. D.; Schubert, U. S. An Amperometric, TemperatureIndependent, and Calibration-Free Method for the Real-Time State-of-Charge Monitoring of Redox Flow Battery Electrolytes. Chem. Mater. 2019, 31, 5363-5369.

(16) Kowalski, J. A.; Fenton Jr., A. M.; Neyhouse, B. J.; Brushett, F. R. A Method for Evaluating Soluble Redox Couple Stability Using Microelectrode Voltammetry. J. Electrochem. Soc. 2020, 167, 160513. 
(17) Neyhouse, B. J.; Tenny, K. M.; Chiang, Y.-M.; Brushett, F. R. Microelectrode-Based Sensor for Measuring Operando Active Species Concentrations in Redox Flow Cells. ACS Appl. Energy Mater. 2021, 4, 13830-13840.

(18) Compton, R. G.; Banks, C. E. Understanding Voltammetry, 2nd ed.; Imperial College Press: London, 2011.

(19) Nichols, J. T.; McLarnon, F. R.; Cairns, E. J. Zinc Electrode Cycle-Life Performance in Alkaline Electrolytes Having Reduced Zinc Species Solubility. Chem. Eng. Commun. 1985, 37, 355-379.

(20) Thomas, S.; Cole, I. S.; Sridhar, M.; Birbilis, N. Revisiting Zinc Passivation in Alkaline Solutions. Electrochimica Acta 2013, 97, 192-201.

(21) Ko, Y.; Park, S.-M. Zinc Oxidation in Dilute Alkaline Solutions Studied by Real-Time Electrochemical Impedance Spectroscopy. J. Phys. Chem. C 2012, 116, 7260-7268.

(22) CRC Handbook of Chemistry and Physics, 102nd ed.; CRC Press: Boca Raton, FL, 2021.

(23) Stamm, J.; Varzi, A.; Latz, A.; Horstmann, B. Modeling Nucleation and Growth of Zinc Oxide during Discharge of Primary Zinc-Air Batteries. J. Power Sources 2017, 360, 136149.

(24) Milshtein, J. D.; Barton, J. L.; Darling, R. M.; Brushett, F. R. 4-Acetamido-2,2,6,6Tetramethylpiperidine-1-Oxyl as a Model Organic Redox Active Compound for Nonaqueous Flow Batteries. J. Power Sources 2016, 327, 151-159. Lei, C.; Yang, F.; Macauley, N.; Spinetta, M.; Purdy, G.; Jankovic, J.; Cullen, D. A.; More, K. L.; Kim, Y. S.; Xu, H. Impact of Catalyst Ink Dispersing Solvent on PEM Fuel Cell Performance and Durability. J. Electrochem. Soc. 2021, 168, 044517. 